\title{
Diversidade genotípica de rotavírus suínos no Estado de São Paulo ${ }^{1}$
}

\author{
Fábio Gregori ${ }^{*}$, César A.R. Rosales ${ }^{3}$ Paulo E. Brandão ${ }^{3}$, Rodrigo M. Soares ${ }^{3}$ \\ e José A. Jerez ${ }^{3}$
}

\begin{abstract}
Gregori F., Rosales C.A.R., Brandão P.E., Soares R.M. \& Jerez J.A. 2009. [Diversity of porcine rotavirus genotypes in São Paulo State, Brazil.] Diversidade genotípica de rotavírus suínos no Estado de São Paulo. Pesquisa Veterinária Brasileira 29(9):707-712. Centro de Pesquisa e Desenvolvimento em Sanidade Animal, Instituto Biológico, Av. Cons. Rodrigues Alves 1252, São Paulo, SP 04014-002, Brazil. E-mail: fabiogregori@biologico.sp.gov.br

Rotavirus is one the most common causes of diarrhea both in humans and different animal species. It was carried out a transversal study with 144 diarrheic fecal samples of piglets, from 16 commercial swine-producing units distributed among 10 municipalities of São Paulo State, Brazil, aiming at the detection of rotavirus occurrence and its molecular characterization according to $G$ and $P$ genotypes. A total of 43 samples $(29.86 \%)$ were positive for rotavirus by Polyacrylamide Gel Electrophoresis (PAGE) and ELISA, in a parallel screening scheme. The nested-multiplex RT-PCR characterization revealed that, separately, the $P[6]$ genotype was the most frequent, detected in $25.58 \%$ of the samples, followed by $P[1](11.63 \%)$ and $P[7](9.3 \%)$. Concomitant infection of the genotypes $P[6]+P[7](9.3 \%), P[1]+P[6](4.65 \%), P[1]+P[6]+P[7](2.33 \%)$ were also found. Similarly, the $\mathrm{G}[5]$ genotype was detected on $30.23 \%$ of the samples, followed by $\mathrm{G}[10]$ (20.93\%), $\mathrm{G}[6](4.65 \%)$ and $\mathrm{G}[5]+\mathrm{G}[10](18.6 \%)$. The genotype G[5]P[6] was the most frequent $(11.63 \%)$, but other combinations and untypeable samples were also observed. Considering the diversity porcine rotavirus found in the surveyed population, specific prophylactic measures should take in charge, for its effectiveness, the cross-protection degree between the genotypes present on vaccine formulations and those that really circulates on a region.
\end{abstract}

INDEX TERMS: Rotavirus, diarrhea, genotypes, porcine, Brazil.

RESUMO.- Rotavírus é uma das causas mais comuns de diarréia tanto em humanos quanto em diferentes espécies animais. Foi conduzido um estudo transversal a partir de 144 amostras fecais diarréicas colhidas de leitões, provenientes de 16 criações comerciais distribuídas por 10 municípios do Estado de São Paulo, Brasil, com o objeti-

\footnotetext{
${ }^{1}$ Recebido em 16 de dezembro de 2008.

Aceito para publicação em 14 de abril de 2009..

${ }^{2}$ Centro de Pesquisa e Desenvolvimento em Sanidade Animal, Instituto Biológico, Av. Cons. Rodrigues Alves 1252, São Paulo, SP 04014002, Brasil. *Autor para correspondência: fabiogregori@ biologico.sp.gov.br

${ }^{3}$ Departamento de Medicina Veterinária Preventiva e Saúde Animal, Faculdade de Medicina Veterinária e Zootecnia, Universidade de São Paulo (USP), Av. Prof. Dr. Orlando Marques de Paiva 87, Cidade Universitária, São Paulo, SP 05508-270.
}

vo de se detectar a ocorrência de rotavírus e realizar sua caracterização molecular quanto seus genotipos $\mathrm{G}$ e $\mathrm{P}$. Um total de 43 amostras $(29,86 \%)$ foram positivas para rotavírus por Eletroforese em Gel de Poliacrilamida (PAGE) e ELISA, num esquema de triagem em paralelo. A caracterização mediante reações do tipo nestedmultiplex RT-PCR demonstrou que, isoladamente, o genotipo $P[6]$ foi o mais frequente, detectado em $25,58 \%$ das amostras, seguido pelo $P[1](11,63 \%)$ e $P[7](9,3 \%)$. Infecções concomitantes de genotipos $P[6]+P[7](9,3 \%)$, $P[1]+P[6](4,65 \%), P[1]+P[6]+P[7](2,33 \%)$ foram também observadas. Analogamente, o genotipo G[5] foi detectado em $30,23 \%$ das amostras, seguido pelo $\mathrm{G}[10]$ (20,93\%) e G[6] $(4,65 \%)$ e G[5]+G[10] (18,6\%). O genotipo G[5]P[6] foi o mais frequente $(11,63 \%)$, porém outras combinações e amostras não tipificáveis também foram observadas. 
Considerando-se a diversidade de rotavírus suínos encontrada na população estudada, medidas profiláticas específicas devem levar em conta, para sua efetividade, o grau de proteção cruzada entre os genotipos presentes nas formulações vacinais e aqueles que realmente são circulantes numa região.

TERMOS DE INDEXAÇÃO: Rotavírus, diarréia, genotipos, suinos, Brasil.

\section{INTRODUÇÃO}

Os rotavírus são um dos principais agentes virais responsáveis pela ocorrência de diarréia em diferentes espécies animais e da gastroenterite infantil (Saif et al. 1994, Estes \& Kapikian 2007). Em suínos, são responsáveis por grandes perdas econômicas, afetando principalmente animais nas fases de maternidade e creche (Will et al. 1994).

Pertencentes à família Reoviridae, o gênero Rotavirus apresenta um genoma composto por 11 segmentos de RNA do tipo dupla-fita, os quais codificam 6 proteínas estruturais (VP1 a VP4, VP6 e VP7) e 6 não-estruturais (NSP1 a NSP6). São atualmente classificados sorologicamente em 5 diferentes grupos ou espécies (A-E) com duas outras possíveis adicionais (F-G), tendo como base a proteína VP6 (Fauquet et al. 2005), sendo os do grupo A os mais prevalentes tanto em humanos quanto em animais (Estes \& Kapikian 2007).

Adicionalmente, os rotavírus do grupo A subdividemse em sorotipos ou genotipos denominados $\mathrm{G}$ e $\mathrm{P}$ de acordo com as características antigênicas ou genéticas apresentadas, respectivamente, pelas proteínas VP7 e VP4 (Dennehy 2008). Estas proteínas também estão envolvidas com a indução de anticorpos neutralizantes e imunidade protetora (Estes \& Kapikian 2007).

Já foram relatados pelo menos 16 diferentes tipos $\mathrm{Ge}$ 27 genotipos $P$ de rotavírus (Parra et al. 2008) sendo que aparentemente há uma predominância de certos genotipos infectando determinadas espécies hospedeiras (Bányai 2005). Sua diversidade genética está associada a diferentes mecanismos de evolução os quais envolvem mutações pontuais (drifts); reestruturações (shifts, reassortants) ou troca de segmentos de RNA entre amostras; e rearranjos (rearrangements), que consistem em duplicações e deleções de sequência nucleotídica dentro de um segmento genômico (Taniguchi \& Urasawa 1995).

A caracterização das amostras circulantes é essencial para o desenvolvimento e utilização de vacinas para rotavírus, já que neste último caso a pressão de seleção exercida pela imunidade vacinal pode levar a emergência de novas amostras (Dennehy 2008).

O potencial zoonótico ou mesmo de transmissão interespécies dos rotavírus já foi descrito em diversos estudos (Cook et al. 2004, Steyer et al. 2008), e particularmente os suínos e bovinos são considerados como importantes reservatórios para a diversidade de amostras humanas (Martella et al. 2007). Nesse sentido, Mascarenhas et al. (2007) consideram uma vigilância simultânea e sequenciamento dos genes de humanos e animais como essenciais para uma melhor compreensão das relações entre amostras que circulam numa mesma região.

O objetivo deste trabalho foi obter a frequência de ocorrência de rotavírus a partir de diferentes criações comerciais de suínos presentes em 10 municípios do Estado de São Paulo, Brasil, e caracterizar as amostras positivas quanto aos seus genotipos $\mathrm{G}$ e $\mathrm{P}$ mediante reações de RT-PCR (transcição reversa - reação em cadeia pela polimerase).

\section{MATERIAL E MÉTODOS}

Um estudo de ocorrência transversal foi conduzido a partir de 144 amostras de fezes de leitões com quadro clínico de diarréia, colhidas em 1999-2001, em 16 granjas comerciais localizadas em 10 municípios do Estado de São Paulo, Brasil, compreendendo as cidades de Bragança Paulista, Campinas, Capivari, Ibiúna, Itaberá, Itú, Pedra Bela, Piedade, Mogi das Cruzes e São José dos Campos.

Foram realizadas suspensões a $20 \%$ das amostras em tampão TRIS-HCl 0,1 M pH 7,3 e clarificadas a $12.000 \mathrm{~g} / 30$ minutos a $4^{\circ} \mathrm{C}$, sendo o sobrenadante armazenado a $-80^{\circ} \mathrm{C}$ até a análise.

Todas as amostras fecais foram triadas em paralelo quanto a presença de rotavírus utilizando-se os testes de Eletroforese em Gel de Poliacrilamida (PAGE) (Herring et al. 1982) e Elisa direto policlonal tipo duplo-sanduíche (Gregori et al. 2000), sendo posteriormente calculados os valores de sensibilidade e especificidade relativas, e valor Kappa (Martin et al. 1987).

A extração de RNA total foi realizada utilizando-se o reagente TRIzol (Invitrogen ${ }^{\mathrm{TM}}$ ) a partir de suspensões fecais clarificadas e de acordo com as instruções do fabricante.

Para a reação de transcrição reversa (síntese de cDNA) foram inicialmente misturados $5,6 \mu \mathrm{L}$ das amostras de RNA extraído com $1,4 \mu \mathrm{L}$ DMSO, desnaturados a $97^{\circ} \mathrm{C}$ por 5 minutos e mantidos em gelo. Estes foram então adicionados à solução composta por 1x First Strand Buffer (Invitrogen ${ }^{\mathrm{TM}}$ ), $1 \mathrm{mM}$ de cada dNTP, $10 \mathrm{mM}$ DTT, $1 \mathrm{pmol} / \mu \mathrm{L}$ de cada iniciador [Con2 e Con3, para a genotipagem P (Gouvea et al. 1994b); Beg9, End9, End9UK e End9CRW8, para a genotipagem G (Gouvea et al. 1994a)] e 200 U M-MLV Reverse Transcriptase (Invitrogen ${ }^{\mathrm{TM}}$ ) para um volume final de reação de $20 \mu \mathrm{L}$, mantidos a $42^{\circ} \mathrm{C}$ por 1 hora.

A seguir, $5 \mu \mathrm{L}$ do DNAc foi adicionado à solução de PCR composta por 1x PCR Buffer (Invitrogen ${ }^{\mathrm{TM}}$ ), 0,2mM de cada dNTP, 0,5 pmol/ $\mu \mathrm{L}$ de cada iniciador [Con2 e Con3, para a genotipagem $P$ (Gouvea et al. 1994b); Beg9, End9, End9UK e End9CRW8, para a genotipagem G (Gouvea et al. 1994a)], $1,5 \mathrm{mM}$ de $\mathrm{MgCl}_{2}, 1,25 \mathrm{U}$ de Taq DNA Polymerase (Invitrogen ${ }^{\mathrm{TM}}$ ), água ultra-pura q.s.p. $50 \mu \mathrm{L}$ e submetidos a 30 ciclos de $94^{\circ} \mathrm{C} / 1$ minuto, $42^{\circ} \mathrm{C} / 2$ minutos, $72^{\circ} \mathrm{C} / 1$ minuto, seguido de $72^{\circ} \mathrm{C} / 10$ minutos para extensão final.

A segunda amplificação (multiplex semi-nested PCR) consistiu em adicionar $5 \mu \mathrm{L}$ de DNA amplificado na etapa anterior à solução composta por 1x PCR Buffer (Invitrogen ${ }^{\mathrm{TM}}$ ), 0,2mM de cada dNTP, 0,5pmol/ $\mu \mathrm{L}$ de cada iniciador [Con2, $\mathrm{pUK}$, pNCDV, pGOTT, pB223 e pOSU para a genotipagem P (Gouvea et al. 1994b); sBeg9, FT5, ET10, DT6, BT11 e HT8 para genotipagem G (Gouvea et al. 1994a)], 1,5mM de $\mathrm{MgCl}_{2}, 1,25$ $\mathrm{U}$ de Taq DNA Polymerase (Invitrogen ${ }^{\mathrm{TM}}$ ), água q.s.p. $50 \mu \mathrm{L}$, e submetidos a 25 ciclos de $94^{\circ} \mathrm{C} / 1$ minuto, $55^{\circ} \mathrm{C} / 2$ minutos, $72^{\circ} \mathrm{C} /$ 
1 minuto, seguindo-se um passo final de $72^{\circ} \mathrm{C} / 10$ minutos para extensão final.

Em seguida, $10 \mu \mathrm{L}$ dos produtos oriundos da $2^{\mathrm{a}}$ amplificação foram analisados por eletroforese em gel de agarose a 1,5\% (p/v) em tampão Tris-borato 0,045 M; EDTA 0,001 M; pH 8,0, fazendo-se corar o gel em banho de água com $0,5 \mu \mathrm{g} / \mathrm{mL}$ de brometo de etídio por 10 minutos. Cada uma das etapas da reação de genotipagem (extração, transcrição reversa, PCR e eletroforese) foi conduzida em salas separadas, utilizando como controle positivo a amostra NCDV de rotavírus e água ultrapura como controle negativo.

\section{RESULTADOS}

Dentre as 144 amostras fecais testadas, obtiveram-se 24 $(16,66 \%)$ amostras positivas para rotavírus por PAGE e $39(27,08 \%)$ amostras positivas por ELISA, totalizando 43 amostras triadas $(29,86 \%)$. Destas, 20 amostras foram positivas em ambas as provas, tendo sido detectada a presença do agente em todos os municípios amostrados. Tomando como referência a prova de PAGE, obteve-se uma sensibilidade relativa do teste de ELISA de $83,33 \%$, especificidade relativa de 84,16\%, e valor Kappa de 0,54.

Os resultados da caracterização genotípica das amostras mediante o emprego da reação de multiplex seminested RT-PCR constam no Quadro 1.

Quadro 1. Distribuição de genotipos $G$ e $P$ de rotavírus mediante o emprego da reação de semi-nested multiplex RT-PCR a partir de material fecal de suínos criados no Estado de São Paulo, Brasil

\begin{tabular}{ccccccc}
\hline Genotipo & $\mathrm{G}[5]$ & $\mathrm{G}[6]$ & $\mathrm{G}[10]$ & $\mathrm{G}[5]+\mathrm{G}[10]$ & $\mathrm{G}[\mathrm{ND}]^{\mathrm{a}}$ & Total \\
\hline $\mathrm{P}[1]$ & 1 & - & 2 & - & 2 & 5 \\
& $(2,33 \%)$ & & $(4,65 \%)$ & & $(4,65 \%)$ & $(11,63 \%)$ \\
$\mathrm{P}[6]$ & 5 & 2 & 1 & 1 & 2 & 11 \\
& $(11,63 \%)$ & $(4,65 \%)$ & $(2,33 \%)$ & $(2,33 \%)$ & $(4,65 \%)$ & $(25,58 \%)$ \\
$\mathrm{P}[7]$ & 1 & - & 1 & 1 & 1 & 4 \\
$\mathrm{P}[1]+\mathrm{P}[6]$ & $(2,33 \%)$ & - & $(2,33 \%)$ & $(2,33 \%)$ & $(2,33 \%)$ & $(9,30 \%)$ \\
$\mathrm{P}[6]+\mathrm{P}[7]$ & - & - & - & 2 & - & 2 \\
& & - & $(2,33 \%)$ & $(6,98 \%)$ & - & $(9,30 \%)$ \\
$\mathrm{P}[1]+\mathrm{P}[6]+\mathrm{P}[7]$ & - & - & - & - & 1 & 1 \\
$\mathrm{P}[\mathrm{ND}]^{\mathrm{a}}$ & 6 & - & 4 & 1 & 5 & 16 \\
& $(13,95 \%)$ & & $(9,30 \%)$ & $(2,33 \%)$ & $(11,63 \%)$ & $(37,21 \%)$ \\
Total & 13 & 2 & 9 & 8 & 11 & 43 \\
& $(30,23 \%)$ & $(4,65 \%)$ & $(20,93 \%)$ & $(18,60 \%)$ & $(25,58 \%)$ & $(100 \%)$
\end{tabular}

$\mathrm{a}[\mathrm{ND}]=$ genotipo não definido.

Observou-se a ocorrência de genotipos G[5], G[6] e $\mathrm{G}[10]$, respectivamente em 21 (48,83\%), 2 (4,65\%) e 17 $(39,53 \%)$ das 43 amostras testadas. Isoladamente, genotipo $\mathrm{G}[5]$ foi o mais frequente, detectado em $30,23 \%$ (13/43) das amostras, seguido pelo G[10] em 20,93\% (9/ 43) e G[6] em 4,65\% (2/43). Houve apenas a ocorrência da combinação de genotipos G[5]+G[10] em 8 amostras $(18,6 \%)$ enquanto que em 11 amostras (25,58\%) não foram definidos os genotipos $\mathrm{G}$.

Definiu-se a ocorrência dos genotipos $\mathrm{P}[1]$, $\mathrm{P}[6]$ e $\mathrm{P}[7]$, respectivamente em $8(18,6 \%), 18(41,86 \%)$, e $9(20,93 \%)$ amostras das 43 testadas. Foram detectadas as seguintes combinações de genotipos $P$ dentre as 43 amostras testadas: $\mathrm{P}[1]+\mathrm{P}[6]$, em 2 amostras (4,65\%); $\mathrm{P}[6]+\mathrm{P}[7]$, em 4 amostras $(9,3 \%)$; e $P[1]+P[6]+P[7]$ em 1 amostra (2,33\%). Em 16 amostras (37,21\%) não foram definidos os genotipos $P$.

Isoladamente, o genotipo $\mathrm{P}[6]$ foi o mais frequente, detectado em 25,58\% (11/43) das amostras, seguido pelo $P[1](11,63 \%=5 / 43)$ e $P[7](9,3 \%=4 / 43)$. Infecções conjuntas de genotipos $P[6]+P[7](9,3 \%), P[1]+P[6](4,65 \%)$, $P[1]+P[6]+P[7](2,33 \%)$ foram também observadas.

A combinação mais frequente foi $\mathrm{G}[5] \mathrm{P}[6]$, presente em 11,63\% (5/43) das amostras fecais. Em 5 amostras $(11,63 \%)$ não foi possível a caracterização em quaisquer genotipos, sendo 2 delas PAGE positivas e ELISA negativas; 1 PAGE e ELISA positiva; e 2 PAGE negativas e ELISA positivas para rotavírus.

\section{DISCUSSÃO}

Considerando-se que todos os 10 municípios envolvidos neste estudo apresentaram pelo menos uma propriedade com amostra positiva para rotavírus através das provas de ELISA e/ou PAGE, aliada a uma frequência de ocorrência de 29,86\%, podemos sugerir uma disseminação relativamente ampla dos rotavírus nas criações suínas do Estado de São Paulo. Estes achados corroboram os de San Juan et al. (1986), Gatti et al. (1989), Alfieri et al. (1994), Rácz et al. (2000), Barreiros et al. (2003), que também demonstraram a ocorrência dos rotavírus nesta mesma espécie animal em diferentes regiões do Brasil.

Com relação à concordância entre PAGE e ELISA, obteve-se o valor de Kappa de 0,54, o que significa uma moderada concordância (Martin et al. 1987), fazendo com que a adoção dos testes em paralelo tenha possibilitado ganhos discretos de sensibilidade diagnóstica durante a triagem de material clínico, sendo os resultados posteriormente confirmados, à exceção de 5 amostras nas quais não puderam ser definidos os genotipos $G$ e $P$, através das reações de semi-nested RT-PCR.

Existem diferentes causas que podem levar a uma indefinição de genotipos mediante a RT-PCR, dentre elas, a circulação de rotavírus pertencentes aos grupos de rotavírus não-A; a presença de genotipos $\mathrm{G}$ e $\mathrm{P}$ não contemplados pelos iniciadores utilizados nas reações ou mesmo mutações pontuais nas amostras circulantes que possam interferir nos seus respectivos sítios de hibridização. Nesse sentido, Simmonds et al. (2008) destacam a importância de uma constante revisão das sequências de primers empregados para a caracterização de rotavírus. Também pode ser considerada a perda de viabilidade da amostra clínica em função do armazenamento, levando a uma degradação da partícula viral particularmente em função de inibidores inespecíficos presentes nas fezes, tal como sugere Rasool et al. (2002).

Aliás, a não caracterização de amostras de rotavírus em seus genotipos é um fato relatado por Winiarczyk et al. (2002), que não obtiveram genotipagem em 3,2\% das 
amostras suínas dos Estados Unidos para G e 12,9\% para $P$. Analogamente, utilizando amostras oriundas da Polônia, não foram definidos os genotipos em $45,5 \%$ delas para $G$ e $61,4 \%$ para $P$. Chan-it et al. (2008) ao submeterem 43 amostras provenientes de criações de suínos na Tailândia à genotipagem, obtiveram um total de $16,27 \%$ de amostras indefinidas quanto ao genotipo $G$ e $18,6 \%$ para $P$. No presente estudo, totalizaram-se 11 (25,58\%) e 16 (37,21\%) das 43 amostras triadas em que não foram obtidas as caracterizações genotípicas $G$ e $P$, respectivamente.

Em suínos, os genotipos G3, G4, G5, G11, P[6] e P[7] são os mais frequentemente detectados, muito embora existam relatos do encontro de amostras G1, G2-like, G6, G8, G9, G10, P[13], P[19], P[23], P[26] e P[27] (Khamrin et al. 2007, Martella et al. 2007, Steyer et al. 2007, Parra et al. 2008).

O genotipo G[5] $(30,23 \%$ do total de amostras genotipadas) foi o de maior ocorrência em infecções singulares, seguido pelo G[10] (20,93\%) e G[6] (4,65\%). Em infecções simultâneas, observou-se somente a associação $G[5]+G[10](18,6 \%)$. Assim sendo, a predominância do genotipo G[5] neste trabalho está em concordância com diversos relatos descritos tanto no Brasil como em outros países.

Com efeito, Rácz et al. (2000) caracterizaram molecularmente amostras fecais de suínos criados na Região Sul do Brasil, detectando genotipos comuns a estas criações, entre eles o G[5], G[3], G[4], bem como G[10] e um atípico, o G[9], e combinações entre G[4] e G[9] e entre $\mathrm{G}[5]$ e G[10]. Santos et al. (1999) utilizando amostras fecais de suínos da região sudoeste do Paraná entre março de 1991 e março de 1992, detectaram os genotipos G[1], G[4], G[5] e G[9]. Ainda neste mesmo Estado, Barreiros et al. (2003) ao investigarem um surto de diarréia em uma criação de suínos observaram a ocorrência de rotavírus G[3] e G[5].

Winiarczyk et al. (2002) detectaram a ocorrência dos genotipos G[3], G[4], G[5], G[9] e G[10] em amostras fecais de suínos nos Estados Unidos e G[3], G[4] e G[5] naquelas oriundas da Polônia, enquanto que Chan-it et al. (2008), detectaram em 6 diferentes granjas da Tailândia, a ocorrência dos genotipos G[3], G[4], G[8] e G[9], sendo que das 7 amostras negativas à genotipagem, concluíram por análise de sequências nucleotídicas que tratavam-se de $\mathrm{G}[3]$ e $\mathrm{G}[5]$.

Os genotipos G[6] e G[10] são predominantemente encontrados em bovinos, descritos nesta espécie tanto no Brasil (Brito et al. 2000, Alfieri et al. 2004) quanto em outros países (Falcone et al. 1999, Garaicoechea et al. 2006, Howe et al. 2008). Além dos relatos anteriormente citados quanto ao encontro destes genotipos em suínos, corroboram os achados de Pongsuwanna et al. (1996) ao terem detectado na Tailândia o sorotipo bovino G10 e os de Martella et al. (2001), que na Itália identificaram em suínos o genotipo G[6]. Torna-se, portanto, necessário o monitoramento de rebanhos bovino e suíno, bem como nas populações humanas, de modo a se detectarem situ- ações de possível infecção cruzada (Martella et al. 2001, Steyer et al. 2008).

Por exemplo, Santos et al. (1998) detectaram em crianças no Estado do Rio de Janeiro, Brasil, a ocorrência de genotipos humanos G[1] e G[3], enquanto que $60 \%$ das infecções eram causadas por genotipos incomuns, entre eles o G[5], G[10] e G[8].

Leite et al. (1996) ao caracterizarem amostras fecais de crianças em 9 Estados do Brasil e do Distrito Federal, referentes ao período de 1982 a 1994, observaram a ocorrência de amostras atípicas, entre elas a G[5] presentes nas caracterizações.

Com relação aos genotipos $\mathrm{P}$ encontrados houve também concordância com aqueles descritos na literatura por outros autores. O genotipo P[6] $(25,58 \%)$ foi o de maior ocorrência em infecções singulares, seguido pelos genotipo $P[1](11,62 \%)$ e $P[7](9,3 \%)$. Observaram-se as associações $P[6]+P[7], P[1]+P[6], E ~ P[1]+P[6]+P[7]$ em $9,3 \%, 4,65 \%$ e $2,32 \%$ das amostras, respectivamente. Ressalta-se, contudo, a participação de genotipo tipicamente bovino $P[1]$ nos achados deste trabalho, o que supõe, aliada a constatação dos genotipos $G$, indicações para possível compartilhamento de amostras entre bovinos e suínos.

Martella et al. (2001) detectaram em suínos, na Itália, o genotipo P[5], tipicamente bovino. Winiarczyk et al. (2002), por sua vez, relatam o encontro dos genotipos $\mathrm{P}[6]$ e P[7] tanto em amostras suínas dos Estados Unidos como da Polônia. Chan-it et al. (2008), na Tailândia, definiram os genotipos $\mathrm{P}[6], \mathrm{P}[7], \mathrm{P}[19]$, além de amostras não tipificáveis.

Rácz et al. (2000) detectaram os genotipos P[6,Gott] e $\mathrm{P}[6, \mathrm{M} 37]$ e $\mathrm{P}$ [7], tanto isoladamente como em associação. Leite et al. (1996) detectaram a predominância dos genotipos $\mathrm{P}[8]$ e $\mathrm{P}[4]$ em crianças no Brasil, porém sendo também detectadas os genotipos incomuns como o P[6] e P[9].

Houve a predominância da ocorrência dos tipos $\mathrm{G}[5] P[6]$ e $G[4] P[6]$, porém foram detectados genotipos atípicos em suínos e reassortants considerando como protótipos as amostras suínas OSU (G5P[7]) e Gottfried (G4P[6]). Recentemente, Steyer et al. (2008) observaram a predominância na Eslovênia, dezesseis diferentes combinações genotípicas, das quais a mais prevalente nesta espécie animal foi G3P[6], G4P[6] e G5P[7].

\section{CONCLUSÕES}

Os resultados demonstram a presença no Estado de São Paulo de genotipos de rotavírus compatíveis com os mais frequentemente descritos em outros países para a espécie suína. Entretanto, observou-se também a presença de genotipos predominantemente bovinos, sugerindo uma potencial infecção cruzada do vírus, o que torna necessário um maior detalhamento da sua circulação entre estas espécies.

Paralelamente, considerando-se a diversidade genotípica de rotavírus encontrada, medidas profiláticas 
baseadas em vacinas devem levar em conta, para sua efetividade, o grau de proteção cruzada entre os genotipos presentes nas formulações vacinais e aqueles de fato circulantes.

Agradecimentos.- À Fundação de Amparo à Pesquisa do Estado de São Paulo (FAPESP) pela concessão de auxílio financeiro (Processo no 99/05912-9) e ao Sr. Alexandre Abelardo Sanches pelo apoio técnico para o desenvolvimento deste trabalho.

\section{REFERÊNCIAS}

Alfieri A.F., Alfieri A.A., Barreiros M.A., Leite J.P. \& Richtzenhain L.J. 2004. $G$ and $P$ genotypes of group $A$ rotavirus strains circulating in calves in Brazil, 1996-1999. Vet. Microbiol. 99(3/4):167-173.

Alfieri A.A., Alfieri A.F., Freitas J.C., Silva C.A., Freire R.L., Arros A.R., Barreiros M.A.B. \& Muller E.E. 1994. Ocorrência de E. coli, Rotavírus, Picobirnavírus e Cryptosporidium em um foco de diarréia do pós-desmame em suínos. Semina, Ciências Agrárias 15(3):5-7.

Bányai K., Forgách P., Erdélyi K., Martella V., Bogdán A., Hocsák E., Havasi V., Melegh B. \& Szücs G. 2005. Identification of the novel lapine rotavirus genotype $P[22]$ from an outbreak of enteritis in a Hungarian rabbitry. J. Virus Res. 113(2):73-80.

Brito W.M.E.D., Munford V., Villaça A.M., Caruzo T.A.R. \& Rácz M.L. 2000. Characterization of mixed infections with different strains of bovine rotavirus in an outbreak of diarrhea in dairy herds in Goiás, Brazil. Braz. J. Microbiol. 31(2):140-145.

Barreiros M.A., Alfieri A.A., Alfieri A.F., Médici K.C. \& Leite J.P. 2003. An outbreak of diarrhoea in one-week-old piglets caused by group $A$ rotavirus genotypes P[7], G3 and P[7], G5. Vet. Res. Commun. 27(6):505-512.

Chan-It W., Khamrin P., Saekhow P., Pantip C., Thongprachum A., Peerakome S., Ushijima H. \& Maneekarn N. 2008. Multiple combinations of P[13]-like genotype with G3, G4, and G5 in porcine rotaviruses. J. Clin. Microbiol. 46(4):1169-1173.

Cook N., Bridger J., Kendall K., Gomara M.I., El-Attar L. \& Gray, J. 2004. The zoonotic potential of rotavirus. J. Infect. 48(4):289-302.

Dennehy P.H. 2008. Rotavirus vaccines: An overview. Clin. Microbiol. Rev. 21(1):198-208.

Estes M.K. \& Kapikian A.Z. 2007. Rotaviruses, p.1917-1974. In: Knipe D.M. et al. (Eds), Fields Virology. 5th ed. Lippincott, Williams and Wilkins, Philadelphia.

Falcone E., Tarantino M., Trani L., Cordioli P., Lavazza A. \& Tollis M. 1999. Determination of bovine rotavirus $G$ and $P$ serotypes in Italy by PCR. J. Clin. Microbiol. 37(12):3879-3882.

Fauquet C.M., Mayo M.A., Maniloff J., Desselberger U. \& Ball L.A. 2005. The Eighth Report of the International Committee on Taxonomy of Viruses. Elsevier Academic Press, San Diego.

Garaicoechea L., Bok K., Jones L.R., Combessies G., Odeón A., Fernandez F. \& Parreño V. 2006. Molecular characterization of bovine rotavirus circulating in beef and dairy herds in Argentina during a 10year period (1994-2003). Vet. Microbiol. 118(1/2):1-11.

Gatti S.M.V., Hara N.H., Ferraz M.M.G. \& Pestana de Castro A.F. 1989. Presence of group $A$ and non-A rotaviruses in neonatal piglets in Campinas, SP, Brazil. Med. Microbiol. Immunol. 178(6):347-349.

Gouvea V., Santos N. \& Timenetsky M.C. 1994a. Identification of bovine and porcine rotavirus $\mathrm{G}$ types by PCR. J. Clin. Microbiol. 32(5):13381340.

Gouvea V., Santos N. \& Timenetsky M.C. 1994b. VP4 typing of bovine and porcine group A rotaviruses by PCR. J. Clin. Microbiol. 32(5):13331337.

Gregori F., Brandão P.E., Rosales C.A.R., Cortez A., Heinemann M.B., Richtzenhain L.J. \& Jerez J.A. 2000. Desenvolvimento de um método de ELISA para a detecção de rotavírus a partir de material fecal. Arqs Inst. Biol., São Paulo, 67(2): 191-194.
Herring A.J., Inglis N.F., Ojeh C.K., Snodgrass D.R. \& Menzies J.D. 1982. Rapid diagnosis of rotavirus infection by direct detection of viral nucleic acid in silver-stained polyacrylamide gels. J. Clin. Microbiol. 16(3):473-477.

Howe L., Sugiarto H. \& Squires R.A. 2008. Use of polymerase chain reaction for the differentiation of Group A bovine rotavirus G6, G8, and G10 genotypes in the North Island Of New Zealand. N. Z. Vet. J. 56(5):218-221.

Khamrin P., Maneekarn N., Peerakome S., Chan-It W., Yagyu F., Okitsu S. \& Ushijima H. 2007. Novel porcine rotavirus of genotype P[27] shares new phylogenetic lineage with G2 porcine rotavirus strain. Virology 361(2):243-252.

Leite J.P.G., Alfieri A.A., Woods P.A., Glass R.I. \& Gentsch J.R. 1996. Rotavirus $G$ and $P$ types circulating in Brazil: Characterization by RTPCR, probe hybridization, and sequence analysis. Arch. Virol. 141(12):2365-2374.

Martella V., Ciarlet M., Bányai K., Lorusso E., Arista S., Lavazza A., Pezzotti G., Decaro N., Cavalli A., Lucente M.S., Corrente M., Elia G., Camero M., Tempesta M. \& Buonavoglia C. 2007. Identification of Group A Porcine Rotavirus Strains Bearing a Novel VP4 (P) Genotype in Italian Swine Herds. J. Clin. Microbiol. 45(2):577-580.

Martella V., Pratelli A., Greco G., Tempesta M., Ferrari M., Losio M.N. \& Buonavoglia C. 2001. Genomic characterization of porcine rotaviruses in Italy. Clin. Diagn. Lab. Immunol. 8(1):129-132.

Martin S.W., Meek A.H. \& Willberg P. 1987. Veterinary Epidemiology. lowa State University Press, Ames.

Mascarenhas J.D.P., Leite J.G., Lima J.C., Heinemann M.B., Oliveira D.S., Araújo I.T., Soares L.S., Gusmão R.P., Gabbay Y.B. \& Linhares A.C. 2007. Detection of a neonatal human rotavirus strain with VP4 and NSP4 genes of porcine origin. J. Med. Microbiol. 56(Pt 4):524532.

Parra G.I., Vidales G., Gomez J.A., Fernandez F.M., Parreño V. \& Bok K. 2008. Phylogenetic analysis of porcine rotavirus in Argentina: Increasing diversity of G4 strains and evidence of interspecies transmission. Vet. Microbiol. 126(1-3):243-250.

Pongsuwanna Y., Taniguchi K., Chiwakul M., Urasawa T., Wakasugi F., Jayavasu C. \& Urasawa S. 1996. Serological and Genomic Characterization of Porcine Rotaviruses in Thailand: Detection of a G10 porcine Rotavirus. J. Clin. Microbiol. 34(5):1050-1057.

Rácz M.L., Kroeff S.S., Munford V., Caruzo T.A.R., Durigon E.L., Hayashi Y., Gouvea V. \& Palombo E. 2000. Molecular characterization of porcine rotaviruses from the southern region of Brazil: Characterization of an Atypical genotype G[9] strain. J. Clin. Microbiol. 38(6):24432446.

Rasool N.B.G., Monroe S.S. \& Glass R.I. 2002. Determination of a universal nucleic acid extraction procedure for PCR detection of gastroenteritis viruses in faecal specimens. J. Virol. Methods 100(1/ 2):1-16.

Saif L.J., Rosen B.I. \& Parwani A.V. 1994. Animal rotaviruses, p.279367. In: Kapikian A.Z. (Ed.), Viral Infections of Gastrointestinal Tract. 2nd ed. Marcel Dekker, New York.

San Juan C.S., Bellinzoni R.C., Mattion N., La Torre J. \& Scodeller E.A. 1986. Incidence of group A and atypical rotaviroses in Brazilian pig herds. Res. Vet. Sci. 41(2):270-272.

Santos N., Lima R.C.C., Nozawa C. M., Linhares R. E. \& Gouvea V. 1999. Detection of porcine rotavirus type G9 and of a mixture of types G1 and G5 associated with Wa-like VP4 specificity: evidence for natural humam-porcine genetic reassortment. J. Clin. Microbiol. 37(8): 2734-2736.

Santos N., Lima R.C.C., Pereira C.F.A. \& Gouvea V. 1998. Detection of rotavirus types $\mathrm{G}[8]$ and $\mathrm{G}[10]$ among Brazilian children with diarrhea. J. Clin. Microbiol. 36(9):2727-2729.

Simmonds M.K., Armah G., Asmah R., Banerjee I., Damanka S., Esona M., Gentsch J.R., Gray J.J., Kirkwood C., Page N. \& Iturriza-Gómara 
M. 2008. New oligonucleotide primers for P-typing of rotavirus strains: strategies for typing previously untypeable strains. J. Clin. Virol. 42(4):368-373.

Steyer A., Poljsak-Prijatelj M., Barlic-Maganja D., Jamnikar U., Mijovski J.Z. \& Marin J. 2007. Molecular characterization of a new porcine rotavirus $\mathrm{P}$ genotype found in an asymptomatic pig in Slovenia. Virology 359(2):275-282.

Steyer A., Poljsak-Prijatelj M., Barlic-Maganja D. \& Marin J. 2008. Human, porcine and bovine rotaviruses in Slovenia: Evidence of interspecies transmission and genome reassortment. J. Gen. Virol. 89(Pt 7):16901698.
Taniguchi K. \& Urasawa S. 1995. Diversity in rotavirus genomes. Semin. Virol. 6(2):123-131.

Will L.A., Paul P.S., Proescholdt T.A., Aktar S.N., Flaming K.P., Janke B.H., Sacks J., Lyoo Y.S., Hill H.T., Hoffman L.J. \& Wu L.L. 1994. Evaluation of rotavirus infection and diarrhea in lowa commercial pigs based on an epidemiologic study of a population represented by diagnostic laboratory cases. J. Vet. Diagn. Invest. 6(4):416-422.

Winiarczyk S., Paul P.S., Mummidi S., Panek R. \& Gradzki Z. 2002. Survey of porcine rotavirus $G$ and $P$ genotype in Poland and the United States using RT-PCR. J. Vet. Med. B, Infect. Dis. Vet. Public Hlth 49(8):373-378. 